\section{Melanome: Austral- asier, Alte und Männer sind am häufigsten betroffen}

Karimkhani C et al. The global burden of melanoma: results from the Global Burden of Disease Study 2015. Br J Dermatol 2017; 177: 134 - 140

Welche Krankheitslast bringen Melanome mit sich? Für eine brauchbare Einschätzung genügen Inzidenz und Mortalität nicht, meint die Global Burden of Disease Study (GBD) - und berechnet „disability-adjusted life years“ (DALYs). Weltweit.

Für 2015 bietet die GBD Schätzungen für 310 Erkrankungen. Eine davon ist das Melanom, die tödlichste aller Hautkrankheiten. Grundlage der Schätzungen sind regionale Erhebungen, wo es sie denn gibt. Wo Daten fehlen, wird interpoliert, hochgerechnet und anhand der Daten aus Nachbarregionen geschätzt.

Die globale Melanom-Inzidenz liegt danach für 2015 bei 351880 Fällen - das entspricht einer altersstandardisierten Rate von 5 Fällen auf 100000 Menschen. Melanome waren verantwortlich für 1 596262 DALYs: verlorene gesunde Lebensjahre - 23 DALYs auf 100000 Menschen, $0,065 \%$ aller DALYs. Melanome waren die Ursache für 59782 Todesfälle - 1 auf 100000 Menschen.

Am meisten Melanom-DALYs finden sich bei den 75- bis-79-Jährigen, gefolgt von den 70- bis-74-Jährigen, danach kommen Menschen ab 80 Jahren. Die Bevorzugung der älteren Jahrgänge ist ein Ergebnis der kumulativen Wirkung von Risikofaktoren wie der UV-Einstrahlung - Ältere hatten einfach mehr Zeit, Sonnenstrahlen zu sammeln.

\section{Australien und Neuseeland vorn}

Die GBD teilt die Welt in 21 Regionen und in denen ist die Häufigkeit von Melanomen höchst unterschiedlich. Mit großem Abstand am meisten betroffen sind Australien und Neuseeland (zusammen: Australasien) mit einer Inzidenzrate von 54, gefolgt von Nordamerika (21), Westeuropa (16), Zentraleuropa (8) und Osteuropa (8). Entsprechend ist die Verteilung für DALYs (Australasien 152, Nordamerika und europäische Regionen 58 66) und Mortalitätsrate (Australasien 6, Nordamerika und Europa 2).

Schaut man die Länder einzeln an, liegen Neuseeland und Australien mit Inzidenzraten von 54 vorn, hinter ihnen folgen Norwegen, Schweden (beide: 26) und die Niederlande (25). Diese Reihenfolge gilt auch für DALYs und die Mortalitätsrate.

Helle Haut zeigt sich hier sehr deutlich als Risikofaktor, besonders wenn sie mit einer kulturellen Wertschätzung von Sonnenbräune zusammenfällt. In Australien und Neuseeland gibt es dann auch noch jede Menge Sonne. Skandinavier tanken die vermutlich eher im Urlaub in südlichen Gefilden und auf Sonnenbänken.

\section{Männer bevorzugt}

Wie in früheren Erhebungen sind Männer häufiger von Melanomen betroffen und sterben auch öfter an ihnen. Die DALYRate liegt für Männer bei 27, für Frauen bei 19. Der Unterschied gilt (fast) weltweit - bis auf die meisten afrikanischen Länder (außer Nordafrika), wo Frauen auf mehr Melanom-DALYs kommen. In allen anderen Ländern sind vermutlich das lückenhaftere Sonnenschutzverhalten der Männer (im Vergleich zu Frauen) und ihre Abneigung gegen Früherkennungsuntersuchungen Gründe für ihre höhere Melanomanfälligkeit.
FAZIT

Die Ergebnisse der GBD zu Melanomen sind wenig überraschend frühere Studienergebnisse werden weitgehend bestätigt. Die GBD betont erneut die dringende Notwendigkeit für (noch) bessere Präventionsmaßnahmen gegen den schwarzen Hautkrebs - vor allem in Ländern mit weitgehend hellhäutiger Bevölkerung. Vielleicht zeigen künftige GDB-Erhebungen (die jetzt jährlich erfolgen sollen) hier erste Erfolge.

Dr. Nina Drexelius, Hamburg 